\title{
Pulmonary amoebiasis presenting as superior vena cava syndrome
}

\author{
A Lichtenstein, A T Kondo, G S Visvesvara, A Fernandez, E F Paiva, T Mauad, M Dolhnikoff, \\ M A Martins
}

\begin{abstract}
Pulmonary amoebiasis without liver involvement occurs sporadically as a result of haematogenous spread from a primary site, the colon. The case history is presented of a patient who developed superior vena cava syndrome due to a pulmonary amoebic abscess without liver involvement. He was initially suspected of having a neoplasm but a combination of tests including histological examination of the H\&E stained excised tissue, immunofluorescence using anti-Entamoeba histolytica antibodies, and serology confirmed the diagnosis of amoebiasis. To our knowledge this is the first description of pulmonary amoebiasis presenting as superior vena cava syndrome.
\end{abstract}

A moebiasis is a protozoan infection caused by Entamoeba histolytica. The prevalence of amoebic colitis and liver abscess is greater in developing regions such as Central and South America than in the industrialised world. It is the third most common cause of death from parasitic diseases after malaria and schistosomiasis. It is estimated that 40-50 million cases of amoebic colitis and liver abscess due to $E$ histolytica occur worldwide and result in 100000 deaths. ${ }^{1-3}$ In Brazil, seroepidemiological studies have shown that infection rates of $E$ histolytica are similar to those in other developing countries. ${ }^{4}$

Pleuropulmonary amoebiasis occurs in 2-3\% of patients with invasive amoebiasis and is frequently associated with liver abscess. Lung disease without liver involvement is exceptional, and it is believed that infection of the lung is a result of haematogenous spread from a primary site-usually the colon.

We report an unusual case of invasive lung amoebiasis that presented as superior vena cava (SVC) syndrome and a brain abscess without liver involvement.

\section{CASE REPORT}

A 31 year old man was clinically diagnosed with ulcerative colitis in 1996 because of intermittent chronic bloody diarrhoea of 7 years' duration. He was treated with sulfasalazine with some improvement in symptoms. In November 1999 he presented with a 2 month history of fever, weight loss, dyspnoea, cough with dark sputum, and haemoptysis. He was treated with several antibiotics with no clinical improvement. A chest radiograph and high resolution CT scan of the thorax revealed a mass in the upper lobe of the right lung (fig $\mathrm{lA}$ and $\mathrm{C}$ ) and he was referred to our service with suspicion of malignancy. The patient was a smoker and had regular contact with captive monkeys.

The patient was admitted with haemoptysis and severe dyspnoea. Blood analyses revealed anaemia (haemoglobin $=8.6 \mathrm{mg} / \mathrm{dl}$ ) and a raised white cell count (50 220 cells/ $\mathrm{mm}^{3}$ ). Serological testing for the human immunodeficiency virus was negative. On the second admission day the patient developed SVC syndrome with swelling of the neck and face and worsening of the dyspnoea. A new chest radiograph and CT scan of the thorax showed enlargement of the mass (fig 1B and D). An abdominal ultrasound scan did not show liver abnormalities and liver enzymes were within normal ranges.

A presumptive diagnosis of SVC syndrome due to a pulmonary neoplasm was made. The patient's clinical condition did not permit a lung biopsy or embolisation of the mass and emergency irradiation of the thorax was administered for 5 days. With the improvement in his clinical condition an open lung biopsy was performed. This resulted in severe bleeding so a right pneumonectomy was carried out. Ten days after surgery the patient developed headache with neck and back stiffness. Cytological analyses of the cerebrospinal fluid (CSF) revealed 1440 cells $/ \mathrm{mm}^{3}$ (79\% neutrophils). Cultures for bacteria and fungi were negative. A cerebral computed tomographic (CT) scan revealed a small lesion in the right caudate nucleus.

Histological examination of the resected right lung showed an irregular cavity $(10 \times 12 \mathrm{~cm})$ containing chocolatecoloured necrotic tissue, with extensive replacement of the pulmonary parenchyma by necrotic tissue and microabscesses and no neoplastic tissue. Periodic acid-Schiff (PAS) and haematoxylin and eosin (H\&E) staining revealed few structures suggestive of amoebae (especially Entamoeba) within the necrotic tissue. Immunofluorescence tests were performed on tissue sections using several different rabbit antisera against $E$ histolytica, Acanthamoeba castellani and Balamuthia mandrillaris. Amoebae in the tissue sections reacted only with the anti-E histolytica serum, producing bright green fluorescence (fig $\mathrm{IE}$ and F). An ELISA test carried out on a serum sample using $E$ histolytica antigens gave a serum IgG titre of 128, suggesting invasive infection due to $E$ histolytica. Three stool samples yielded negative results for the identification of amoeba.

Treatment with ceftriaxone and metronidazole was administered for 21 days and the patient showed rapid clinical improvement. A total colonoscopy performed 1 month after the specific treatment was completed showed small aftoid ulcers in the colon and rectum. Colon biopsies revealed chronic non-specific colitis with eosinophils but no microorganisms. During a 2 year follow up period the patient had no intestinal or neurological symptoms and remained in good health.

\section{DISCUSSION}

To our knowledge, there are no reports of pulmonary amoebiasis presenting as SVC syndrome. Previous reports of non-neoplastic SVC syndrome include Klebsiella, ${ }^{5}$ Nocardia, ${ }^{6}$ Mycobacteria, ${ }^{7}$ and other sporadic aetiological agents. Patients with a condition similar to the case described are frequently suspected of having a malignancy at the time of the initial investigation, resulting in a delay in the correct diagnosis. 

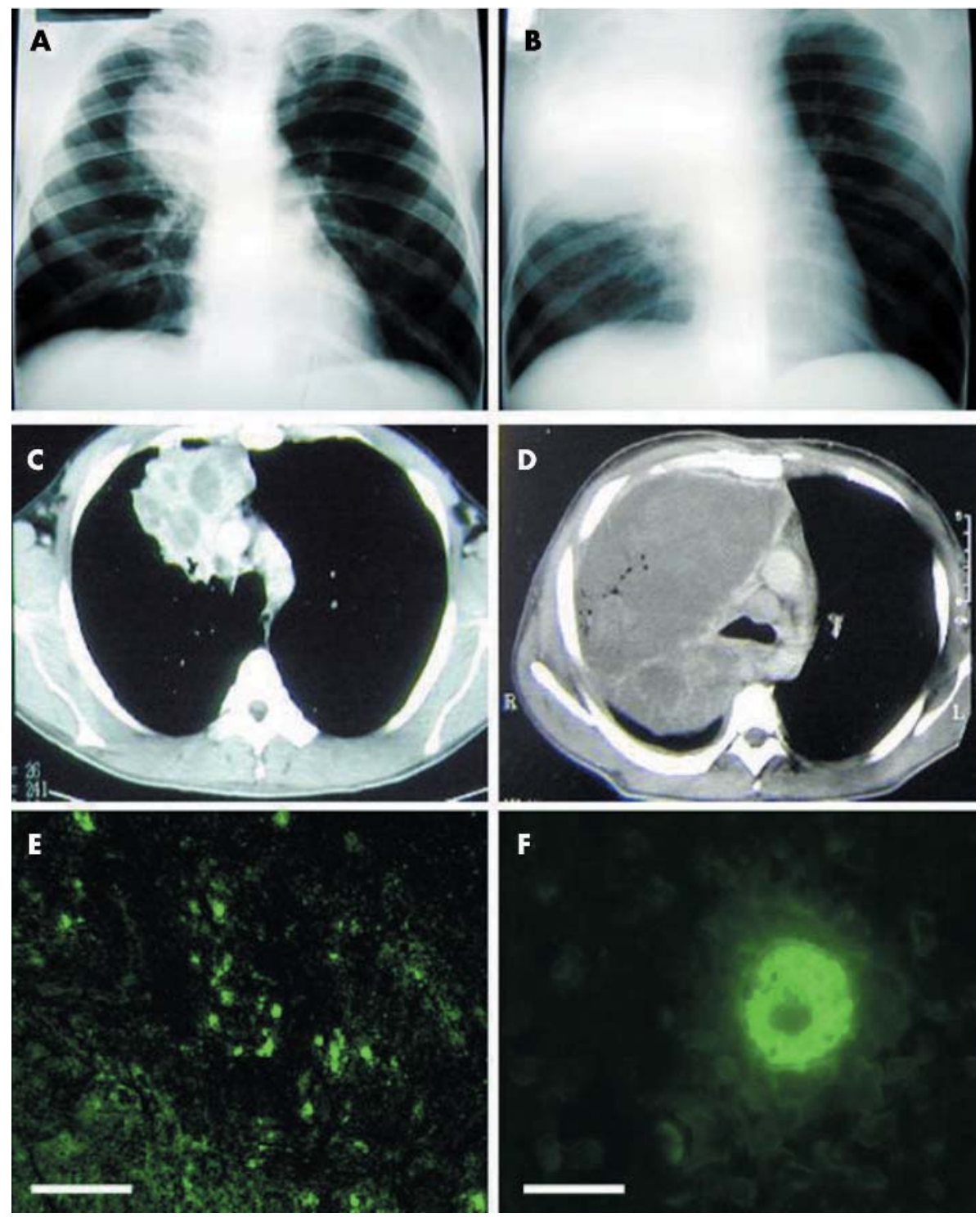

Figure 1 Postero-anterior radiographs of the thorax taken (A) on admission showing a large mass in the superior lobe of the right lung and (B) at second admission day showing significant enlargement of the mass compared with (A). High resolution computed tomographic (HRCT) scans of the thorax taken (C) on admission showing the pulmonary mass in the right lung and (D) at second admission day showing significant enlargement of the mass. (E) Lung section showing necrotic tissue which reacted with anti-E histolytica antibodies in the immunofluorescence assay (note a small number of amoebae with bright apple green fluorescence: scale bar $=250 \mu \mathrm{m}$ ). (F) High power view of a lung section reacted with anti- $E$ histolytica antibodies in the immunofluorescence assay showing a single amoeba with bright apple green fluorescence (scale bar $=25 \mu \mathrm{m}$ ).

The most frequent extra-intestinal site of involvement in amoebiasis is the liver (3-9\% of all cases). Amoebic liver abscess is about 10 times more common in men than in women. ${ }^{3}$ The lung is involved far less often, being affected usually by the extension of liver abscesses $(6-40 \%$ of patients with amoebic liver abscess). ${ }^{3}$ The lower and middle lobes of the right lung are mostly affected in the form of empyema, abscess and hepatobronchial fistula. Like liver abscess, pulmonary amoebiasis is about 10 times more common in young men aged $20-40$ years than in women. ${ }^{1}$ Pulmonary amoebiasis is generally confounded with bacterial pneumonia resulting in prolonged illness of the patient and eventually death. ${ }^{8}$ The mortality rate in one case series of 501 patients was $11.4 \%{ }^{9}$

In our patient we believe that lung and brain involvement occurred secondary to haematogenous spread from the colon. It is estimated that amoebic lung disease without liver involvement occurs in $14.3 \%$ of all cases with lung involvement by amoeba, and there are sporadic case reports showing this type of appearance. ${ }^{10}$ Our patient had been previously diagnosed with inflammatory bowel disease. The colonoscopy showed lesions suggestive of amoebiasis in the rectum and colon, but amoeba micro-organisms were not seen in colonic biopsy specimens. However, we cannot exclude the possibility that the patient had intestinal amoebiasis, since intestinal symptoms disappeared after specific treatment. Furthermore, biopsies were performed after the specific treatment, possibly explaining the negative findings. A combination of ulcerative colitis and the amoebic carrier state has also been described. ${ }^{11}$ Although trophozoites were not found in the $\mathrm{CSF}$, treatment with metronidazole was effective in healing the brain lesion and improving symptoms.

Another interesting point is that our patient had regular contact with captive monkeys. It is known that non-human primate species are infected with $E$ histolytica. ${ }^{12}$ Captive monkeys may therefore serve as carriers of $E$ histolytica and 
it is possible that the monkeys may have been the source of infection in our patient.

Lung amoebiasis is a life threatening but treatable condition. We recommend that $E$ histolytica should be included as a possible cause in the differential diagnosis of lung lesions in young patients, especially in countries where amoebiasis is endemic.

\section{Authors' affiliations}

A Lichtenstein, A T Kondo, E F Paiva, M A Martins, Department of Medicine, São Paulo University Medical School, São Paulo, Brazil T Mauad, M Dolhnikoff, Department of Pathology, São Paulo University Medical School, São Paulo, Brazil

G S Visvesvara, Division of Parasitic Diseases, National Centers for Infectious Diseases, Centers for Disease Control and Prevention, Atlanta, Georgia, USA

A Fernandez, Department of Thoracic Surgery, São Paulo University Medical School, São Paulo, Brazil

Correspondence to: Dr T Mauad, Department of Pathology, Faculdade de Medicina da Universidade de Sao Paulo, Av. Dr. Arnaldo 455 1st floor, 01246-903 São Paulo SP, Brazil; tmauad@usp.br

Received 6 January 2004

Accepted 28 March 2004

\section{REFERENCES}

1 Shamsuzzaman SM, Hashiguchi Y. Thoracic amebiasis. Clin Chest Med 2002;23:479-92.

2 Anon. WHO/PAHO/UNESCO report of a consultation of experts on amebiasis. Weekly Epidemiological Report of the World Health Organization 1997;72:97-9.

3 Petri AW Jr, Singh U. Diagnosis and management of amebiasis. Clin Infect Dis 1999;29:1117-25.

4 Braga LL, Lima AA, Sears CL, et al. Seroepidemiology of Entamoeba histolytica in a slum in northeastern Brazil. Am J Trop Med Hyg 1996;55:693-7.

$5 \mathrm{Kim}$ JY, Lim CM, Koh Y, et al. A case of superior vena cava syndrome caused by Klebsiella pneumoniae. Eur Respir J 1997;10:2902-3.

6 Abdelkafi S, Dubail D, Bosschaerts T, et al. Superior vena cava syndrome associated with Nocardia farcinica infection. Thorax 1997;52:492-3.

7 Minguez C, Roca B, Gonzalez-Mino C, et al. Superior vena cava syndrome during the treatment of pulmonary tuberculosis in an HIV-1 infected patient. $J$ Infect 2000;40:187-9.

8 Stanley SL Jr. Amoebiasis. Lancet 2003;361:1025-34.

9 Ibarra-Perez C. Thoracic complications of amebic abscess of the liver. Chest 1981;79:672-7.

10 Afsar S, Choudhri AN, Ali J, et al. Primary pulmonary amoebiasis: an unusual cause of pulmonary consolidation. J Pak Med Assoc 1992;42:245-6.

11 Dunzendorfer T, Kasznica J. Amebic and/or ulcerative colitis. Gastrointest Endosc 1998;48:450-1.

12 Marquez-Monter H, Fuentes-Orozco R, Correa-Lemus I, et al. Invasive amebiasis in a spider monkey (Ateles geoffroyi). Case report and a review of the literature of amebiasis in non human primates. Arch Invest Med (Mex) 1992;22:75-8. 